\title{
Geometric Optimization of the 56MHz SRF Cavity and its Frequency Table
}

Xiangyun Chang, Ilan Ben-Zvi

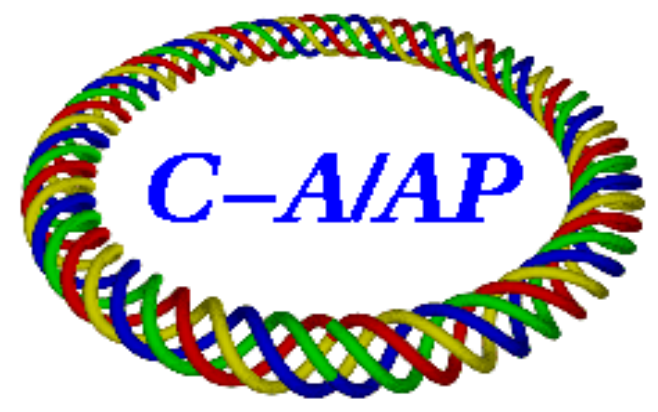

\section{Collider-Accelerator Department Brookhaven National Laboratory Upton, NY 11973}

Notice: This document has been authorized by employees of Brookhaven Science Associates, LLC under Contract No. DE-AC02-98CH10886 with the U.S. Department of Energy. The United States Government retains a nonexclusive, paid-up, irrevocable, world-wide license to publish or reproduce the published form of this document, or allow others to do so, for United States Government purposes. 
Geometric optimization of the 56MHz SRF cavity and its frequency table

\author{
Xiangyun Chang, Ilan Ben-Zvi
}

September 24, 2008

\begin{abstract}
It is essential to know the frequency of a Superconducting Radio Frequency (SRF) cavity at its "just being fabricated" stage because frequency is the key parameter in constructing the cavity. In this paper, we report our work on assessing it. We can estimate the frequency change from stage to stage theoretically and/or by simulation. At the operating stage, the frequency can be calculated accurately, and, from this value, we obtain the frequencies at other stages. They are listed in a table that serves to check the processes from stage to stage. Equally important is optimizing the geometric shape of the SRF cavity so that the peak electric-field and peak magnetic-field are as low as possible. It is particularly desirable in the $56 \mathrm{MHz}$ SRF cavity of RHIC to maximize the frequency sensitivity of the slow tuner. After undertaking such optimization, our resultant peak electric-field is only $35.3 \mathrm{MV} / \mathrm{m}$, and the peak magnetic-field is $840 \mathrm{G}$ at $2 \mathrm{MV}$ of voltage across the cavity gap. To quench superconductivity in an SRF cavity, it is reported that the limit of the peak magnetic-field is 1800G [1], and that of the peak electric-field is more than $100 \mathrm{MV} / \mathrm{m}$ for a SRF cavity [2]. Naturally, for the complex geometry of this cavity (including numerous electron beam welds) and $4.2 \mathrm{~K}$ operations we do not expect record field performance, but a useful $2 \mathrm{MV}$ gap voltage is reasonable to expect. Our simulations employed the codes Superfish and Microwave Studio.
\end{abstract}

\title{
1 Introduction
}

The function of the 56MHz SRF cavity is to provide a large longitudinal separatrix (or "bucket") for stored bunches of the RHIC ion beam. Hence, the voltage across the cavity is a critical parameter for its use. The higher the voltage, the larger is the bucket. RHIC applications require that the voltage is about $2 \mathrm{MV}$ or better. However, voltage is limited by the peak magnetic- and electric-fields that a superconducting cavity can reach. Optimizing the cavity shape to lower the peak electric and field magnetic field becomes important. Another important parameter must be considered simultaneously, viz., the frequency sensitivity to the slow tuner's movement. Figure 1 shows the slow tuner. We require as high sensitivity as achievable, such that the slow tuner, with its limited physical-adjustment range, covers as much as possible of the frequency range. An increase in the slow tuner's sensitivity raises the frequency tolerance of the cavity. In this paper, we describe our studies to satisfy these criteria. 


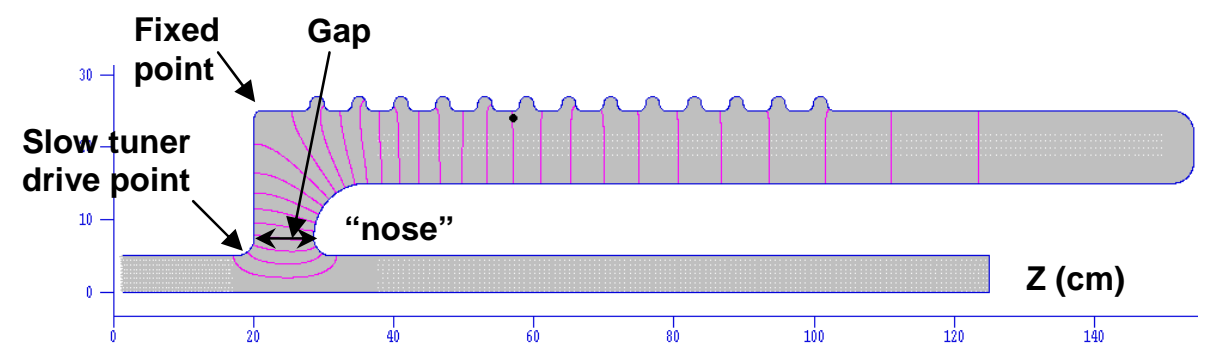

Fig.1 Geometry of the 56MHz SRF cavity

The maximum peak electric field can be lowered by decreasing the curvature of the surface at the location of the peak field, while the slow tuner's sensitivity is increased by decreasing the gap shown in Fig.1. Accordingly, we aimed to optimize the shape of the "nose" and the gap to reduce the ratio of the peak field to the accelerating field, and also increase the slow tuner's frequency sensitivity.

SRF cavities experience different physical processes, from their primary fabrication stage to their final operating stage. The $56 \mathrm{MHz}$ cavity has a very high $\mathrm{Q}$ and is essentially beam driven. In normal operation it is detuned a few hundred hertz from the beam drive frequency. Thus its operating frequency controls the voltage on the gap and must be controlled very accurately $(\sim 1 \mathrm{~Hz})$. The slow tuner's elastic range is about $\pm 9 \mathrm{kHz}$ around the operating point, and therefore, the frequency error of the fabrication stage should be smaller than this range. Thus, it is essential to know accurately the frequency of the cavity at its different fabrication, assembly and operational stages. We chose to define seven stages from initially fabricating the cavity to operating it, not all necessarily in the order given: 1 . The cavity is fabricated, down to one last weld, to a specific frequency : 2 . The final weld is made. 3 . The HOM dampers are installed; 4 . The cavity is chemically cleaned (leading to some material loss); 5 . the cavity is pumped down; 6. The cavity is cooled down to liquid helium temperature; and, 7, the slow tuner is adjusted to the operating point. The frequency varies in each of these stages. The changes are assessed either theoretically or by simulation. We can calculate the frequency at the operating stage accurately and so precisely derive the frequency at each stage.

\section{The operating frequency}

RHIC's circumference is $3833.845 \mathrm{~m}$. At a beam energy of $\gamma=100$, the bunch revolution frequency is $78.19238076 \mathrm{kHz}$. Since there are 120 beam bunches in the ring (with some buckets empty), the bunch frequency is $9.3830856912 \mathrm{MHz}$. Then, the 56MHz cavity's frequency as a sub-harmonic is $56.298514 \mathrm{MHz}$. Similarly, we calculate that the beam frequency is $56.30133 \mathrm{MHz}$ for $\gamma=\infty$, and $56.30088 \mathrm{MHz}$ for $\gamma=250$. The change in beam frequency is only $2.4 \mathrm{kHz}$ from $\gamma=100$ to $\gamma=\infty$. For our optimization, we will assume that the beam frequency is $56.2985 \mathrm{MHz}$. The cavity's resonance frequency has to approach this frequency, but stay below it by an amount that depends on the beam current and the required voltage, or about 200 Hertz.

3 Frequency change from stage to stage 
To bring the cavity to stage 1 , the joint which forms the last weld is machined, and the cavity is clamped without a weld and its frequency is measured under controlled conditions of temperature and humidity as described below. The temperature assumed is $293 \mathrm{~K}$, thus for any deviation from this temperature the frequency has to be corrected by $0.4 \mathrm{kHz} / \mathrm{K}$. The sign of the correction is to increase the target frequency for an increase in temperature. Likewise, the assumed humidity is $40 \%$. The target frequency should be increased by $0.56 \mathrm{kHz}$ per $10 \%$ increase of the relative humidity. Once the target frequency is reached, we move to perform the weld. In stage 2 the cavity's last weld has been done, leading to some weld shrinkage. The weld point is near the "fixed point" in fig. 1. The shrinkage is estimated to be $0.6 \pm 0.06 \mathrm{~mm}$. This number will be eventually measured experimentally. The frequency change per mm of shrinkage is $-23 \mathrm{kHz}$ by simulation. Negative sign indicates decrease of the frequency. So, in this stage the frequency change is $-13.8 \pm 1.4 \mathrm{kHz}$.

The HOM dampers are installed from stage 2 to stage 3. Simulation by Microwave Studio yielded a frequency change in this step of $+3 \mathrm{kHz} /$ damper. Since we are going to use 4 dampers, the overall frequency will increase $12 \mathrm{kHz}$. We used the Microwave Studio to do this calculation because of the lack of axial asymmetry of the HOM dampers. The error in this step is within $1 \mathrm{kHz}$.

At stage 4 the cavity is cleaned chemically, and it is assumed that there is a 200 um thick material loss throughout the inside of the cavity. Simulation gives a frequency change of $-15 \mathrm{kHz}$ per mm of material loss (Superfish), so the frequency change in this step is 3 $\mathrm{kHz}$. The error in this step is estimated to be $\sim 0.5 \mathrm{kHz}$ due to the uncertainty of the material loss thickness.

There are three effects on the cavity's frequency going to stage 5 where the air is pumped out. One reflects the deformation of the cavity due to the change in pressure. The cavity structure is well designed and we found that the frequency change due to the deformation is only $-0.28 \mathrm{~Hz}$ per mbar decrease of pressure in the cavity. Hence, the total frequency change from air to vacuum is $0.28 \pm 0.01 \mathrm{kHz}$. The second effect is from the change of permittivity from air $\left(\varepsilon_{\mathrm{r}(\mathrm{air})}=1.00054\right)$ to vacuum $\left(\varepsilon_{\mathrm{r}(\mathrm{vac})}=1\right)$. This is estimated as follows:

$f_{\text {Vac }} / f_{\text {Air }}=\sqrt{\varepsilon_{r}(\text { air })} \approx 1.00027$.

That causes an increase in frequency of $15.2 \pm 0.3 \mathrm{kHz}$. The third effect is due to the air humidity. It is found by simulation that the frequency increases $0.56 \mathrm{kHz}$ per $10 \%$ increase of the relative humidity. The standard air condition is $20 \%$ relative humidity and the relative humidity in a room with air conditioning is about $40 \%$. So the frequency will increase about $1.1 \mathrm{kHz}$.

The cavity is cooled down to the temperature of liquid helium at stage 6 , during which the cavity shrinks due to the thermal-expansion effect. We calculated the integrated thermal-expansion coefficient from room temperature $(293 \mathrm{~K})$ to liquid-helium temperature $(4.2 \mathrm{~K}$ or $2 \mathrm{~K})$. The coefficient is found to be 0.00143 . Therefore, the frequency change in this step is $80.5 \mathrm{kHz}$. The thermal expansion coefficient is $7.3 \times 10^{-6}$ $/ \mathrm{K} @$ room temperature. This corresponds $0.4 \mathrm{kHz} / \mathrm{K}$. So a controlled temperature is necessary during the frequency measurement step of the fabrication process (stage 1). The error in this step is estimated to be $0.5 \mathrm{kHz}$.

At the last stage, the slow tuner is in a position to let the beam current excite the cavity to its desired voltage. This position is deeper than the tuner's free-standing state. During this 
last step, the slow tuner is pulled out and passes through its free-standing state (FSS). The frequency increases to approach the operating point from below. Simulation (Superfish) give a value of $17 \mathrm{kHz} / \mathrm{mm}$ for the frequency sensitivity due to the slow tuner's movement, assuming that the distance between the free-standing state to the operating point is $1.5 \mathrm{~mm}$. So, the frequency change in this step is $25.5 \mathrm{kHz}$. The error is estimated to be $0.5 \mathrm{kHz}$ in this step. The slow tuner is pushed in $1.5 \mathrm{~mm}$ from the FSS during the energy ramping up. This can make the detune frequency be more than $50 \mathrm{kHz}$.

\section{The frequency table}

Table 1, below, is the frequency table of the $56 \mathrm{MHz}$ SRF cavity derived from the above analysis. The table is ordered in inverse sequence of the stages described above, since we start at a known frequency for operations and work back to determine the required frequency during the last weld.

Table 1

\begin{tabular}{|c|c|c|c|c|}
\hline Stage & Status & $\begin{array}{l}\text { Frequency } \\
{[\mathrm{MHz}]}\end{array}$ & $\begin{array}{l}\text { Frequency } \\
\text { change }[\mathrm{kHz}]\end{array}$ & Estimated error \\
\hline 7 & Operational position & 56.2985 & & 0 \\
\hline 6 & Tuner moved to FSS & 56.2730 & $\begin{array}{l}-25.5 \\
(17 \mathrm{kHz} / \mathrm{mm})\end{array}$ & 0.5 \\
\hline 5 & $\begin{array}{l}\text { Warm up to R.T. } \\
\text { (expansion) }\end{array}$ & 56.1925 & $\begin{array}{l}-80.5 \\
(\alpha=0.00143 \\
4.2 \mathrm{k} \sim 293 \mathrm{k})\end{array}$ & $\begin{array}{l}0.5 \\
(7.3 \mathrm{E}-6 / \mathrm{K}, \\
0.4 \mathrm{kHz} / \mathrm{K} @ 273 \mathrm{~K})\end{array}$ \\
\hline 4 & $\begin{array}{l}\text { Fill in air (pressure } \\
\text { def.) }\end{array}$ & 56.1928 & +0.28 & 0.01 \\
\hline 4 & $\begin{array}{l}\text { Fill in air } \\
\text { (Permittivity change) }\end{array}$ & 56.1776 & $\begin{array}{l}-15.2 \\
(\varepsilon=1.00054)\end{array}$ & 0.3 \\
\hline 4 & $\begin{array}{l}\text { Fill in air } \\
\text { (Humidity difference) }\end{array}$ & 56.1787 & $\begin{array}{l}+1.1 \\
\text { (@40\% } \\
\text { humidity) }\end{array}$ & 0.1 \\
\hline 3 & $\begin{array}{l}\text { Undo the effect of } \\
\text { chemistry }\end{array}$ & 56.1817 & $\begin{array}{l}+3.0 \\
(-15 \mathrm{kHz} / \mathrm{mm})\end{array}$ & 0.5 \\
\hline 2 & $\begin{array}{l}\text { Remove } 4 \text { HOM } \\
\text { dampers (As } \\
\text { fabricated) }\end{array}$ & 56.1697 & $\begin{array}{l}-12 \\
(3 \mathrm{kHz} \\
\text { /damper }) \\
\end{array}$ & 1 \\
\hline 1 & $\begin{array}{l}\text { Before welding the } \\
\text { last piece }\end{array}$ & 56.1835 & $\begin{array}{l}+13.8 \mathrm{kHz} \\
(-23 \mathrm{kHz} / \mathrm{mm})\end{array}$ & 1.4 \\
\hline
\end{tabular}

It should be pointed that the fundamental damper produces a large frequency change during operations, but eventually it is completely withdrawn before the cavity is excited to full voltage. The error for each step is given. The total frequency error is about $2.5 \mathrm{kHz}$ from the table. The plastic adjusting range of the slow tuner is about $\pm 3 \mathrm{~mm}$. This corresponds to $\pm 50 \mathrm{kHz}$ of overall frequency tolerance. The elastic range of the slow 
tuner during operation is assumed to be from $+0.5 \mathrm{~mm}$ to $+1.5 \mathrm{~mm}$ from FSS. This range covers the ion beam energy of $\gamma=50$ to $\gamma=\infty$.

The parameters listed in the table may vary depending on the final configuration. For example, frequency change due to HOM damper may vary due to its geometry change.

Although the opening of the holes for dampers lowers the cavity frequency for about 100 $\mathrm{kHz}$, it only affects the initial cavity geometry calculation.

\section{Gap optimization}

To optimize the cavity's geometry, we first explored the accuracy of the simulations. Fig. 2 gives the results of simulations of the peak field a $56 \mathrm{MHz}$ cavity with the same geometry but with various mesh sizes. It reveals that the simulations converge when the mesh is smaller than $1 \mathrm{~mm}$. We used a $0.5 \mathrm{~mm}$ mesh in our optimization.

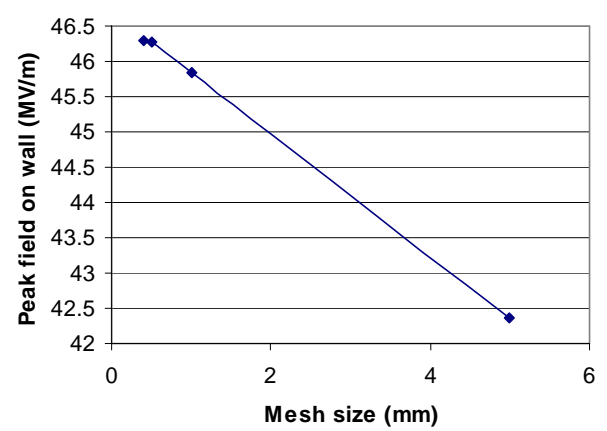

Fig.2 Peak-field results for the cavity with various mesh size

Our next step was to optimize the gap. In principle, keeping the voltage unchanged, then the smaller the gap is, the higher is the peak electric-field. . On the other hand, the smaller the gap becomes, the higher is the slow tuner's frequency sensitivity due to the increased capacitance. Fig. 3 shows that the peak field does not change much when the gap size is above $8.5 \mathrm{~cm}$. Fig. 4 illustrates the rapid increase in the slow tuner's frequency sensitivity when the size of the gap is falling. We chose a gap of $8.5 \mathrm{~cm}$.

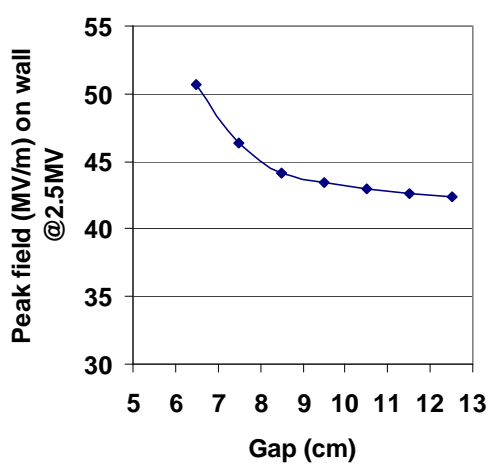

Fig.3 Peak electric-field on the cavity's wall as a function of the gap's size 


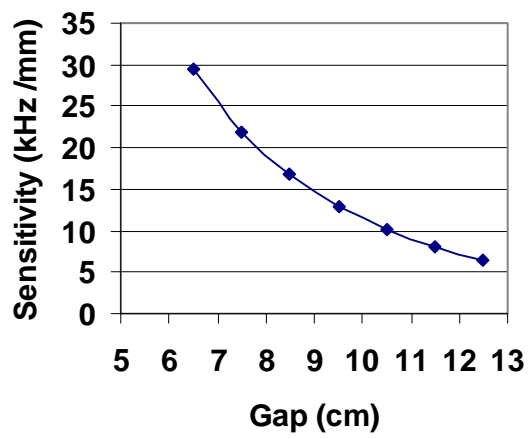

Fig.4 Slow tuner's sensitivity as a function of the gap's size

6. Optimization of the shape of the "nose"

Figure 5 depicts the shape of the "nose". In our optimization, we kept R1+R2 a constant. We first optimized the shape assuming that the upper half of the nose is an ellipse; the optimized result demonstrated that the shape actually is a circle.

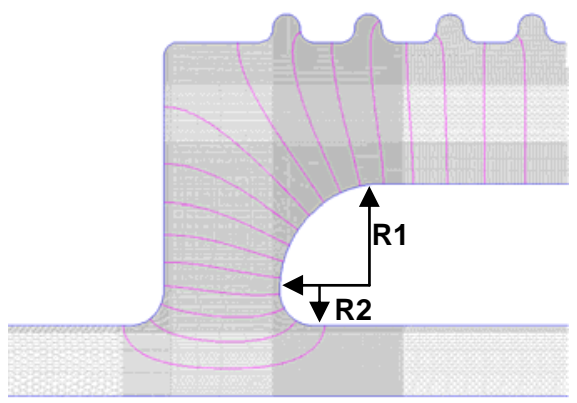

Fig.5 Peak electric-field vs. gap size

Fig. 6 plots the peak electric-field as a function of the size of the radius, revealing that the optimized point is $\mathrm{R} 2=2.4 \mathrm{~cm}$. However, this does not constitute a big difference to $\mathrm{R} 2=2.5 \mathrm{~cm}$ that we selected.

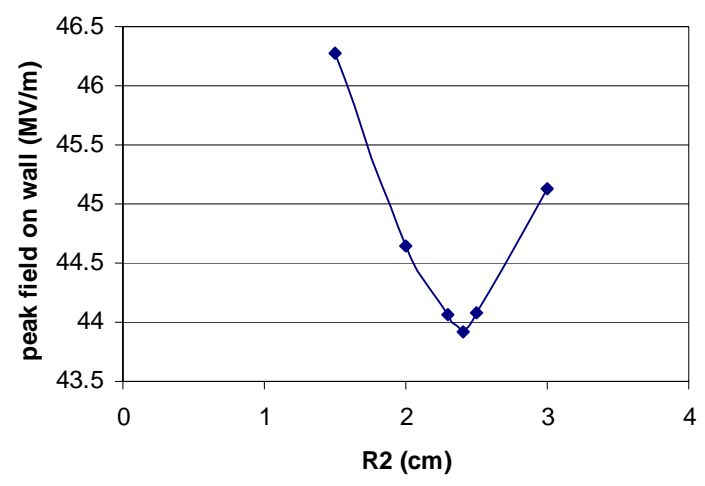

Fig.6 Peak electric-field as a function of the radius R2

The following lists some parameters of our optimized cavity.

Frequency

$$
=56.29903 \mathrm{MHz}
$$


Stored energy

Operating temperature

Power dissipation

Q

Shunt impedance

$\mathrm{r} / \mathrm{Q}$

mum $H($ at $Z, R=151.27,14.97)=83483.4 \mathrm{~A} / \mathrm{m}$

Maximum $E($ at $Z, R=30.12,12.15)=44.09 \mathrm{MV} / \mathrm{m}$
$=214.43$ Joules

$=4.2000 \mathrm{~K}$

$=42.23 \mathrm{~W}$

$=1.796 \mathrm{E}+9$

$=74002 \mathrm{MOhm} / \mathrm{m}$

$=81.95 \mathrm{Ohm}$

\section{Conclusion}

We generated a frequency table by simulations and calculations. The overall error is about $2.5 \mathrm{kHz}$. The plastic adjusting range of the slow tuner covers $50 \mathrm{kHz}$ frequency error. The elastic adjusting range of the slow tuner covers the ion beam energy of $\gamma=50$ to $\gamma=\infty$. At 293K, 1ATM, $40 \%$ humidity, 200um material loss in chemistry, before the last piece is welded, the frequency should be $56.1835 \mathrm{MHz}$.

We optimized the cavity gap and the nose shape to achieve a low peak electric-field, and low peak magnetic-field, and a slow tuner with high frequency sensitivity. The resulting gap is $8.5 \mathrm{~cm}$, and the radii for the nose are $7.5 \mathrm{~cm}$ for its upper part, and $2.5 \mathrm{~cm}$ for its lower part. The peak electric field is $44.1 \mathrm{MV} / \mathrm{m}$ at voltage of $2.5 \mathrm{MV}$, and the slow tuner's frequency-sensitivity is $17 \mathrm{kHz} / \mathrm{mm}$.

\section{References:}

[1] K. Saito, Proc. of the PAC.03, p.462, Portland, Oregon, USA, May12-16, 2003

[2] P. Kneisel, "LATEST DEVELOPMENTS IN SUPERCONDUCTING RF STRUCTURES FOR BETA=1

PARTICLE ACCELERATION”, proc. of EPAC06, Edinburgh, Scotland, July, 2006 